\title{
Accuracy of Microwave Cavity Perturbation Measurements
}

\author{
R.G. Carter, Member. IEEE
}

\begin{abstract}
Techniques based on the perturbation of cavity resonators are commonly used to measure the permittivity and permeability of samples of dielectric and ferrite materials at microwave frequencies. They are also used to measure the local electric and magnetic field strengths in microwave structures including the shunt impedances of cavity resonators and the coupling impedances of slow-wave structures. This paper re-examines the assumptions made in the theory of these techniques and provides estimates of the errors of measurement arising from them.
\end{abstract}

\section{Introduction}

When a small object is introduced into a microwave cavity resonator the resonant frequency is perturbed $[1,2]$. Because it is possible to measure the change in frequency with high accuracy this provides a valuable method for measuring the electric and magnetic properties of the object if the properties of the cavity are known, or for characterising the cavity if the properties of the perturber are known. Techniques based upon this principle are in common use for measuring the dielectric and magnetic properties of materials at microwave frequencies [3]. They also used for measuring the local electric and magnetic field strengths within microwave structures and, especially, for finding the shunt impedances of cavity resonators for use in klystrons and particle accelerators and the coupling impedances of slow-wave structures for use in travelling-wave tubes and linear accelerators $[4,5,6]$. The theoretical basis of these measurements is well-established but involves some simplifications. This paper re-examines these assumptions and approximations to show the effect which they have on the accuracy of the measurements.

\footnotetext{
${ }^{1}$ R.G. Carter is with the Engineering Department, Lancaster University, Lancaster LA1 4YR, U.K.
} 


\section{Theory}

The theory of the perturbation of cavity resonators has been given by a number of authors. The treatment given here is essentially that presented by Waldron [1] but with some differences which maintain the symmetry of the equations. We shall study the properties of two identical cavity resonators containing non-conducting perturbing objects. Let the fields in the two cavities be $E_{0} \exp j \omega_{0} t$ and $H_{0} \exp j \omega_{0} t$ and $E_{1} \exp j \omega_{1} t$ and $H_{1} \exp j \omega_{1} t$. Making use of Maxwell's curl equations we obtain

$$
\begin{aligned}
& \nabla \times E_{0}=-j \omega_{0} B_{0} \\
& \nabla \times H_{1}=j \omega_{1} D_{1}
\end{aligned}
$$

Taking the scalar product of $H_{1}$ with eq. 1 and $E_{0}$ with eq. 2 and subtracting gives

$$
H_{1} \cdot\left(\nabla \times E_{0}\right)-E_{0} \cdot\left(\nabla \times H_{1}\right)=-j \omega_{0} H_{1} \cdot B_{0}-j \omega_{1} E_{0} \cdot D_{1}
$$

But

$$
\nabla \cdot\left(E_{0} \times H_{1}\right)=H_{1} \cdot\left(\nabla \times E_{0}\right)-E_{0} \cdot\left(\nabla \times H_{1}\right)
$$

Therefore

$$
\nabla \cdot\left(E_{0} \times H_{1}\right)=-j \omega_{0} H_{1} \cdot B_{0}-j \omega_{1} E_{0} \cdot D_{1}
$$

Integrating eq. 5 over the volume of the cavity, and making use of Gauss' Theorem

$$
\iiint \nabla \cdot A d v=\iint A \cdot d S
$$

yields

$$
\iint_{S}\left(E_{0} \times H_{1}\right) \cdot d S=\iiint_{V}\left(-j \omega_{0} H_{1} \cdot B_{0}-j \omega_{1} E_{0} \cdot D_{1}\right) d v
$$

Where $\mathrm{S}$ is the surface of the cavity and $\mathrm{V}$ its volume. By a similar argument, exchanging the subscripts, we obtain

$$
\iint_{S}\left(E_{1} \times H_{0}\right) \cdot d S=\iiint_{V}\left(-j \omega_{1} H_{0} \cdot B_{1}-j \omega_{0} E_{1} \cdot D_{0}\right) d v
$$


If the walls of the cavity can be regarded as perfectly conducting then $E$ is normal to the wall and $\boldsymbol{H}$ is tangential to the wall. The vector products are thus tangential to the wall and the left hand sides of equations 7 and 8 are zero. Equating the right hand sides of eqs. 7 and 8 and rearranging gives

$$
j \omega_{0} \iiint_{V}\left(E_{1} \cdot D_{0}-H_{1} \cdot B_{0}\right) d v=j \omega_{1} \iiint_{V}\left(E_{0} \cdot D_{1}-H_{0} \cdot B_{1}\right) d v
$$

If we now assume that the cavity with subscript 0 is empty and let $\omega_{1}=\omega_{0}+\Delta \omega$ eq. 9 can be re-arranged to give

$$
\frac{\Delta \omega}{\omega_{0}}=\frac{\iiint_{V}\left[\left(E_{1} \cdot D_{0}-E_{0} \cdot D_{1}\right)-\left(H_{1} \cdot B_{0}-H_{0} \cdot B_{1}\right)\right] d v}{\iiint_{V}\left(E_{0} \cdot D_{1}-H_{0} \cdot B_{1}\right) d v}
$$

The integrand in the numerator of this equation is zero everywhere outside the volume of the perturbing object. We may therefore restrict the volume of integration to the volume of the object denoted by $V_{1}$. Thus

$$
\frac{\Delta \omega}{\omega_{0}}=\frac{\iiint_{V_{1}}\left(E_{1} \cdot D_{0}-E_{0} \cdot D_{1}\right)-\left(H_{1} \cdot B_{0}-H_{0} \cdot B_{1}\right) d v}{\iiint_{V}\left(E_{0} \cdot D_{1}-H_{0} \cdot B_{1}\right) d v}
$$

The only assumption which has been made so far is that the cavity walls are perfectly conducting. There is no restriction on the size or shape of the perturbing object, or of its material provided that it is not conducting. The symmetry of eq. 11 ensures that its validity is independent of the magnitudes of the fields in the two cavities. For a non-magnetic object the second bracket in the numerator of eq. 11 is zero and

$$
\frac{\Delta \omega}{\omega_{0}}=\frac{\iiint_{V_{1}}\left(E_{1} \cdot D_{0}-E_{0} \cdot D_{1}\right) d v}{\iiint_{V}\left(E_{0} \cdot D_{1}-H_{0} \cdot B_{1}\right) d v}
$$

If we set $\boldsymbol{E}_{1}=\boldsymbol{E}_{0}+\mathbf{e}$ and similarly for the other variables eq. 11 becomes 


$$
\frac{\Delta \omega}{\omega_{0}}=\frac{\iiint_{V_{1}}\left(e \cdot D_{0}-E_{0} \cdot d\right)-\left(h \cdot B_{0}-H_{0} \cdot b\right) d v}{\iiint_{V}\left(E_{0} \cdot D_{0}-H_{0} \cdot B_{0}\right)+\left(E_{0} \cdot d-H_{0} \cdot b\right) d v}
$$

Equations 11,12 and 13 cannot be applied directly because it is not normally possible to find closed-form expressions for the fields in the perturbed cavity. In order to derive useful formulae certain approximations must be made.

Assumption 1: $\quad$ The perturber is made of homogeneous isotropic material so that $D$ and $B$ can be expressed in terms of $E, H$ and the permittivity and permeability of the material. Equation 11 becomes

$$
\frac{\Delta \omega}{\omega_{0}}=\frac{\iiint_{V_{1}}\left[\epsilon_{0}\left(1-\epsilon_{r}\right) E_{0} \cdot E_{1}-\mu_{0}\left(1-\mu_{r}\right) H_{0} \cdot H_{1}\right] d v}{\iiint_{V}\left(E_{0} \cdot D_{1}-H_{0} \cdot B_{1}\right) d v}
$$

Assumption 2: The perturbation is small so that the second term in the denominator of eq. 13 can be neglected. Equation 14 becomes

$$
\frac{\Delta \omega}{\omega_{0}}=\frac{\iiint_{V_{1}}\left[\epsilon_{0}\left(1-\epsilon_{r}\right) E_{0} \cdot E_{1}-\mu_{0}\left(1-\mu_{r}\right) H_{0} \cdot H_{1}\right] d v}{\iiint_{V}\left(E_{0} \cdot D_{0}-H_{0} \cdot B_{0}\right) d v}
$$

This assumption has removed the symmetry of the equation so that the frequency perturbation is dependent on the relative amplitudes of the fields in the empty and perturbed cavities. The denominator is recognised as $4 W_{0}$ where $W_{0}$ is the the stored energy in the empty cavity.

Assumption 3: $\quad$ The perturber is small enough for $E$ and $\boldsymbol{H}$ to be effectively constant within it so that the numerator is equal to the integrand multiplied by the volume of the perturber. Equation 15 becomes 


$$
\frac{\Delta \omega}{\omega_{0}}=\frac{\left[\epsilon_{0}\left(1-\epsilon_{r}\right) E_{0} \cdot E_{1}-\mu_{0}\left(1-\mu_{r}\right) H_{0} \cdot H_{1}\right] V_{1}}{4 W_{0}}
$$

Assumption 4: $\quad$ The $E$ and $\boldsymbol{H}$ fields outside the perturber are unchanged by its presence and those within the perturber can be determined from the boundary conditions at its surface. This enables simple expressions for the frequency perturbation to be derived in two cases:

a) Long thin cylindrical dielectric rod aligned parallel to $E_{0}$ :

Since the tangential electric field is continuous at the surface of the rod $(r=b)$ it follows that $E_{1}=E_{0}$ and, since $\mu_{\mathrm{r}}=1$, equation 16 reduces to the usual approximate formula for perturbation of the frequency by a thin dielectric rod:

$$
\frac{\Delta \omega}{\omega_{0}}=\frac{\epsilon_{0}\left(1-\epsilon_{r}\right)\left|E_{00}\right|^{2} \pi b^{2} L}{4 W_{0}}
$$

where $E_{00}$ is the magnitude of $E_{0}$ on the axis and $L$ is the length of the rod.

b) Dielectric sphere:

Under the quasi-static approximation the electric field within a dielectric sphere placed in a uniform external electric field $\boldsymbol{E}_{0}$ is given by [7]

$$
E_{1}=\frac{3 E_{0}}{\epsilon_{r}+2}
$$

Substitution of this expression into equation 16 and taking $\mu_{\mathrm{r}}=1$ yields the usual expression for the perturbation of the frequency by a small dielectric sphere:

$$
\frac{\Delta \omega}{\omega}=-\frac{\pi R^{3} \epsilon_{0}\left|E_{0}\right|^{2}}{W_{0}} \cdot\left\{\frac{\epsilon_{r}-1}{\epsilon_{r}+2}\right\}
$$

where $R$ is the radius of the sphere. 
It is generally assumed that these approximate expressions are accurate enough for most purposes but the range of validity of the assumptions has not been checked. In the sections which follow we examine this problem by comparing the approximate solutions with those obtained by direct application of eq. 12.

\section{Perturbation of a pill-box cavity by a dielectric rod}

Consider a pill-box cavity, excited in the $\mathrm{TM}_{100}$ mode, which is perturbed by a cylindrical dielectric rod placed along its axis as shown in fig. $1 \mathrm{a}$. The general solutions for the electric field inside and outside the rod are

$$
\begin{gathered}
E_{1}=E_{z}=A J_{0}\left(\sqrt{\epsilon_{r}} k_{1} r\right) \\
E_{1}=E_{z}=B J_{0}\left(k_{1} r\right)+C Y_{0}\left(k_{1} r\right)
\end{gathered}
$$

where $A, B$ and $C$ are constants, $J_{0}$ and $Y_{0}$ are the Bessel functions of the first and second kinds and $k_{1}=\omega_{1} / c$ where $\mathrm{c}$ is the velocity of light in vacuum. We can choose $A=1$ without loss of generality. The constants $B$ and $C$ are determined by requiring that $E_{z}$ and $\partial E_{z} / \partial r$ are continuous at the surface of the rod so that

$$
\begin{gathered}
B=\frac{Y_{0}\left(k_{1} a\right) J_{0}\left(\sqrt{\epsilon_{r}} k_{1} b\right)}{J_{0}\left(k_{1} b\right) Y_{0}\left(k_{1} a\right)-J_{0}\left(k_{1} a\right) Y_{0}\left(k_{1} b\right)} \\
C=-B \cdot J_{0}\left(k_{1} a\right) / Y_{0}\left(k_{1} a\right)
\end{gathered}
$$

The requirement that $E_{\mathrm{z}}$ is zero at $r=a$ yields the determinantal equation

$$
\sqrt{\epsilon_{r}} \cdot \frac{J_{1}\left(\sqrt{\epsilon_{r}} k_{1} b\right)}{J_{0}\left(\sqrt{\epsilon_{r}} k_{1} b\right)}=\frac{J_{1}\left(k_{1} b\right) Y_{0}\left(k_{1} a\right)-J_{0}\left(k_{1} a\right) Y_{1}\left(k_{1} b\right)}{J_{0}\left(k_{1} b\right) Y_{0}\left(k_{1} a\right)-J_{0}\left(k_{1} a\right) Y_{0}\left(k_{1} b\right)}
$$

This equation can be solved numerically ${ }^{2}$ to obtain $k_{1}$ and $\omega_{1}$ for given values of $a, b$ and $\varepsilon_{\mathrm{r}}$.

2 The results presented in this paper were obtained using Mathcad $_{\mathbb{8}} 8$. 
For the empty cavity we note that

$$
\omega_{0}=k_{0} c=2.405(c / a)
$$

Since the solutions scale directly with the dimensions we can display the ratio $\omega_{1} / \omega_{0}$ against bia for various values of $\varepsilon_{\mathrm{r}}$ as shown in fig. 2 .

In the empty cavity the electric field is given by

$$
E_{0}=E_{z}=J_{0}\left(k_{0} r\right)
$$

and the magnetic field by

$$
H_{0}=H_{\theta}=j \sqrt{\frac{\epsilon_{0}}{\mu_{0}}} J_{1}\left(k_{0} r\right)
$$

In the perturbed cavity the magnetic field is:

$$
H_{1}=H_{\theta}=j \sqrt{\epsilon_{r}} \sqrt{\frac{\epsilon_{0}}{\mu_{0}}} J_{1}\left(\sqrt{\epsilon_{r}} k_{0} r\right)
$$

inside the rod, and

$$
H_{1}=H_{\theta}=j \sqrt{\frac{\epsilon_{0}}{\mu_{0}}}\left[B J_{1}\left(k_{1} r\right)+C Y_{1}\left(k_{1} r\right)\right]
$$

outside the rod.

When the fields defined by these equations are substituted into eq. 12 the results are identical to those obtained from eq. 25 . 
The stored energy in the empty cavity is given by

$$
W_{0}=\pi \epsilon_{0} L \int_{0}^{a} J_{0}\left(k_{0} r\right)^{2} r d r
$$

Substituting this expression into eq. 17 , and noting that $E_{00}=1$, we obtain

$$
\frac{\Delta \omega}{\omega_{0}}=1.856\left(1-\epsilon_{r}\right)(b / a)^{2}
$$

The frequency ratios computed from eq. 32 for relative permittivities of 2,5 and 10 are compared with the exact results in figure 2. It is seen that there is good agreement between the two sets of results if $b / a \leq 0.1$ and that the agreement deteriorates as $b / a$ increases and as the relative permittivity increases. The accuracy is revealed more clearly in figures $3 a$ and $b$ which show the error in the approximate solutions for the ranges $0<b / a<0.1$ and $0<b / a$ $<0.2$ respectively. If the normalised rod diameter is less than 0.1 the approximate solution is accurate to better than $1 \%$ for $\varepsilon_{\mathrm{r}} \leq 10$. If $\varepsilon_{\mathrm{r}}=2$ the difference between the exact and approximate formulae is negligible. But these results conceal possible sources of error which make it unwise to assume that the same accuracies will apply to other shapes of the perturbing object.

Figure 4 shows comparisons between the numerators and denominators of the exact and approximate expressions (equations 12 and 17). From these it is clear that the apparent accuracy of eq.17 is a consequence of the balancing of approximately equal errors in the numerator and the denominator. These errors lie in the range $1 \%-30 \%$ for the cases investigated. Thus the assumption that the second term in the denominator of eq. 13 can be neglected is not as valid as has been generally supposed. The physical explanation of this result is that the electric field within the rod is over-estimated by assumption 3 since the radial variation of the field within the rod has been neglected. The field outside the rod is reduced by the presence of the rod so that assumption 2 causes the denominator to be overestimated. It is fortuitous that the errors compensate each other in this case but it is not safe to assume that a similar cancellation will occur in other cases. It is therefore possible that measurements made using perturbation methods may be in error by several percent. 
One of the main uses of this theory is to determine the relative permittivities of samples of dielectric material in the form of rods. Since the method relies on the frequency perturbation caused by the rod it is sensitive to quite small errors in the value of the perturbed frequency. This is illustrated in fig. 5 in which values of the frequency perturbation obtained from the exact theory have been used to obtain the relative permittivity from eq. 32 . It can be seen that appreciable errors occur when the relative permittivity is calculated by the approximate method.

\section{Perturbation of a pill-box cavity by a dielectric sphere}

When a dielectric sphere is place in a uniform electric field the field within the sphere is given by eq. 18 and the additional electric field components outside the sphere produced by the polarisation of the sphere are [7]:

$$
\begin{aligned}
& E_{\rho}=E_{0}\left(\frac{\epsilon_{r}-1}{\epsilon_{r}+2}\right) R^{3}\left(\frac{2 \cos \phi}{\rho^{3}}\right) \\
& E_{\phi}=E_{0}\left(\frac{\epsilon_{r}-1}{\epsilon_{r}+2}\right) R^{3}\left(\frac{\sin \phi}{\rho^{3}}\right)
\end{aligned}
$$

in spherical polar co-ordinates.

We will assume that the dielectric sphere is placed on the axis of a pill-box cavity as shown in fig. $1 \mathrm{~b}$. In order to be able to compute the frequency perturbation from eq. 12 it is necessary to make two assumptions:

Assumption 5: The sphere is small enough for the field in which it is placed to be effectively constant. If we require the variation of the field to be not more than $1 \%$ over the space occupied by the sphere then $k_{0} R=0.2$ and thus $\mathrm{R} / \mathrm{a} \leq 0.083$. For a $5 \%$ field variation $\mathrm{R} / \mathrm{a} \leq 0.19$. 
Assumption 6: $\quad$ The sphere is small enough for the perturbation of the external field to be effectively zero on the boundary of the cavity. If we set a limit of $1 \%$ on the perturbation then $R / a$ and $2 R / L \leq 0.2$. Thus for most cavities the second condition will be satisfied whenever the first condition is true.

The field components outside the sphere are given, in cylindrical polar co-ordinates, by:

$$
\begin{gathered}
E_{1 z}=E_{0 z}+E_{\rho} \cos \phi-E_{\phi} \sin \phi \\
H_{1 \theta}=\frac{-j}{\mu_{0} \omega_{1}} \frac{\partial E_{1 z}}{\partial r}
\end{gathered}
$$

where $\omega_{1}$ is the, as yet unknown, perturbed frequency. The remaining field components are not required because their inner products with the unperturbed field components are zero. Within the sphere, for consistency, we must take $E_{0}=E_{00}$ and $H_{1 \theta}=0$. Equation 12 can then be evaluated numerically to obtain values for the frequency perturbation which are exact for small spheres. Figure 6 shows how the ratio of the perturbed to the unperturbed frequency depends upon the radius and the relative permittivity of the sphere as found from the approximate and exact calculations. Since we have used the same expression for the electric field inside the sphere for both the approximate and exact calculations it follows that fig. 6 shows the effect of neglecting the second term in the denominator of eq.13 in this case. The error introduced by this assumption is much less than in the case of perturbation by a rod because of the much smaller change in the fields outside the perturber.

Perturbation measurements using a dielectric sphere are commonly used to determine the electric field distribution within a microwave structure. By substituting the frequency perturbation computed from eq. 12 into eq. 19 we can find the error in the determination of the field. The results of this calculation in fig. 7 show that the error is less than $1 \%$ for typical sizes of sphere.

\section{Conclusions}

The results presented in this paper have shown that the assumptions made in the approximate 
theory of the perturbation of cavities by dielectric objects are not always valid. In particular we have seen that the figures for the relative permittivity of dielectric rods may be in error by $5 \%$ for typical rod sizes. If the method is used to find the relative permittivity of rods having a uniform, but non-circular, cross-section it is likely that similar accuracies will be obtained. When perturbation methods are used to characterise cavity resonators and other microwave structures it is likely that the relative permittivity of the perturber will have been obtained by a perturbation measurement. In that case the errors in measurement should be small provided that the same assumptions were made in interpreting both measurements and that the assumption that the perturber is located in a region of uniform electric field is satisfied to a good approximation.

\section{References}

1. Waldron, R.A., Perturbation theory of resonant cavities, Procedings IEE, Vol.107C, pp.272-274, September 1960

2. Kraszewski, A.W., and Nelson, S.O., Observations on resoriant cavity perturbation by dielectric objects, IEEE Transactions on Microwave Theory and Techniques, Vo.40, pp.151-155, January 1992

3. Altschuler, H.M., Dielectric Constant in Handbook of Microwave Measurements (3rd edn.), Sucher, M. and Fox, J. (eds), Polytechnic Institute of Brooklyn Press (1963)

4. Horsley, A.W. and Pearson, A., Measurement of dispersion and interaction impedance characteristics of slow-wave structures by resonance methods, IEEE Transactions on Electron Devices, Vol.13, pp.962-969, December 1966

5. Connolly, D., Determination of the interaction impedance of coupled-cavity slow-wave structures, IEEE Transactions on Electron Devices, Vol.23, pp.491-493, May 1976

6. Hanna, S.M., Bowden, G.B., Hoag, H.A., Loewen, R.J., Miller, R.L., Ruth, R.D. and Wang, J.W., Microwave cold-testing techniques for the NLC, Proceedings of the European Particle Accelerator Conference (1996)

7. Bleaney, B.I. and Bleaney, B., Electricity and Magnetism, Oxford Univerisity Press (1957) 


\section{Captions for figures}

Fig.1 Pill-box cavity resonator perturbed by (a) a dielectric rod, and (b) a dielectric sphere.

Fig.2 Comparison of the resonant frequency of a pill-box cavity, perturbed by a dielectric rod, computed by exact and approximate methods.

Fig.3 Error in the resonant frequency of a pill-box cavity, perturbed by a dielectric rod, computed by the approximate method.

Fig.4 Comparison between the numerators and denominators of the exact and approximate formulae for computing the resonant frequency of a pill-box cavity, perturbed by a dielectric rod.

Fig.5 Error in the calculation of the relative permittivity of a dielectric rod using the approximate formula.

Fig.6 Comparison of the resonant frequency of a pill-box cavity, perturbed by a dielectric sphere, computed by exact and approximate methods.

Fig.7 Error in the magnitude of the electric field in a pill-box cavity, perturbed by a dielectric sphere, computed by the approximate method. 


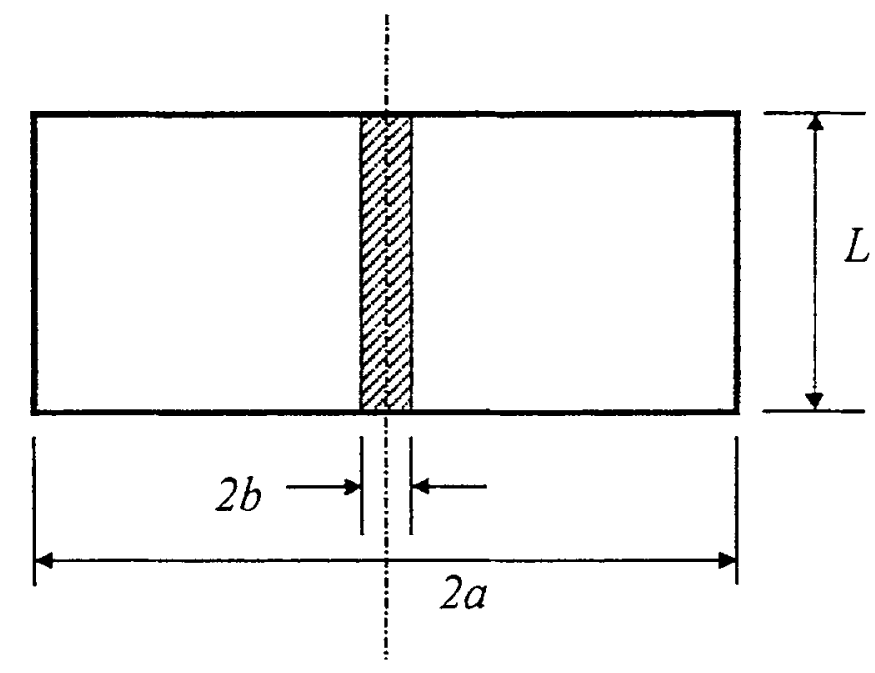

(a)

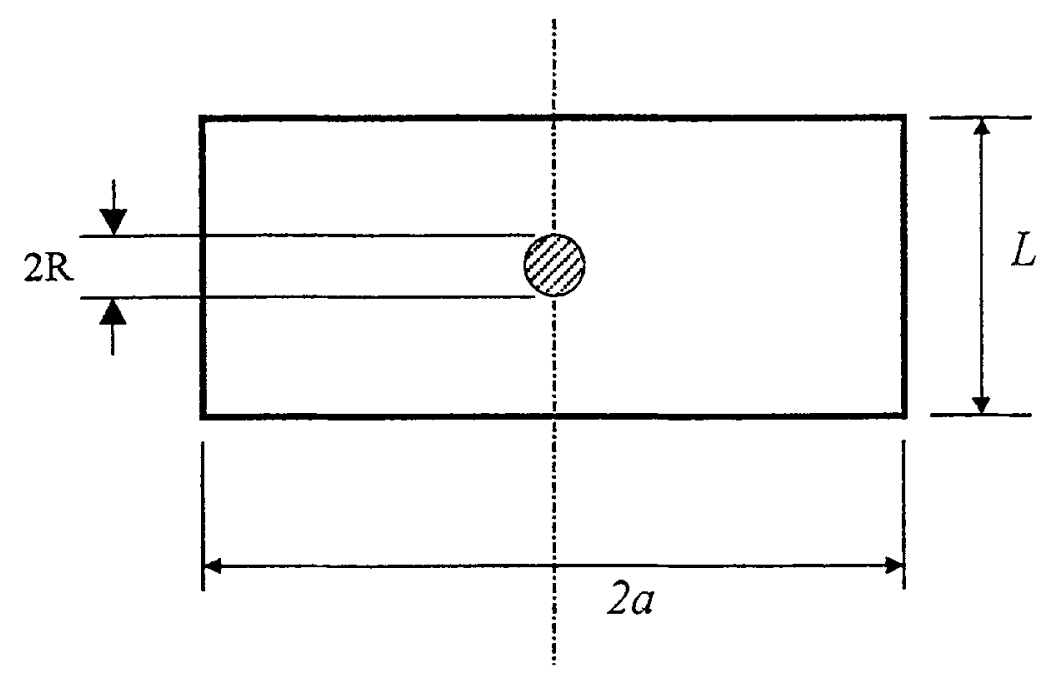

(b)

Fig.1 


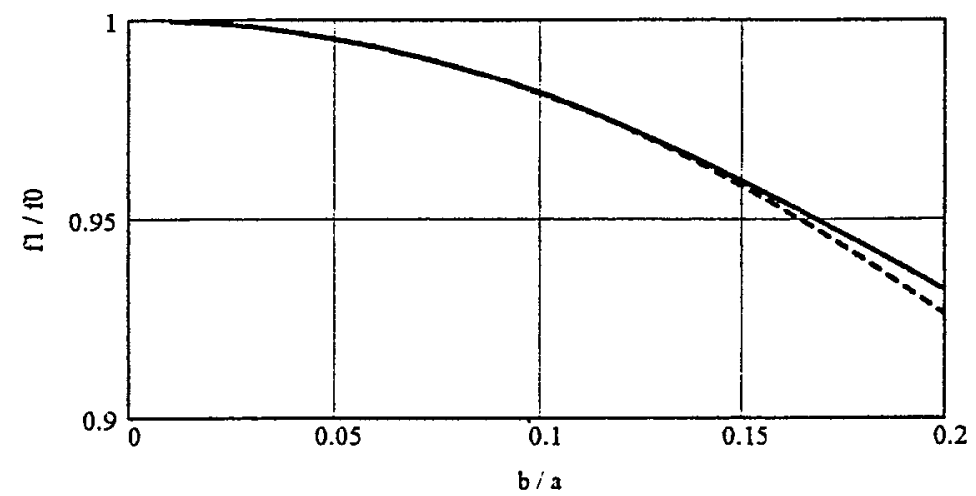

Exact

Approximate

(a) $\mathrm{Epsr}=2$

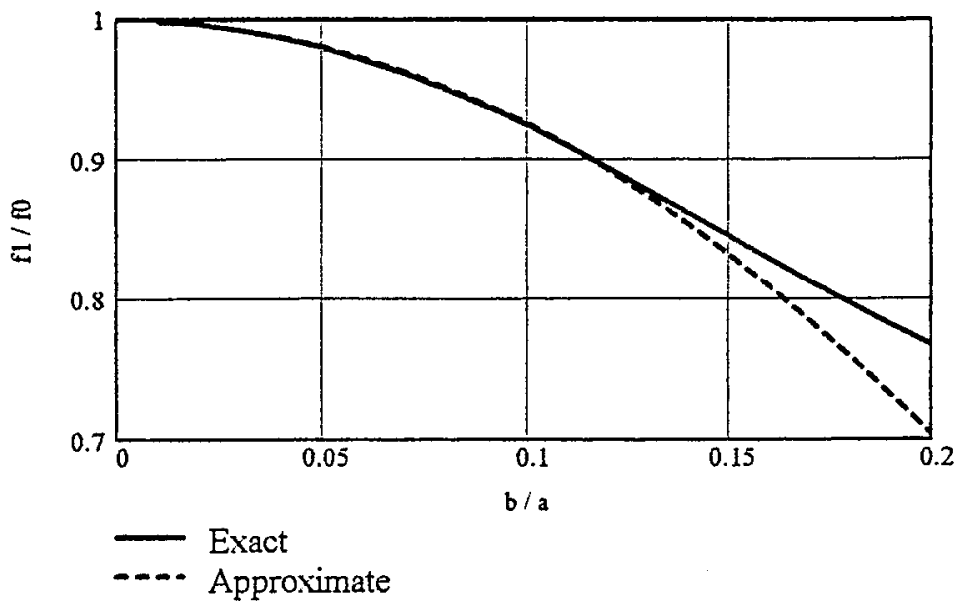

(b) $\mathrm{Epsr}=5$

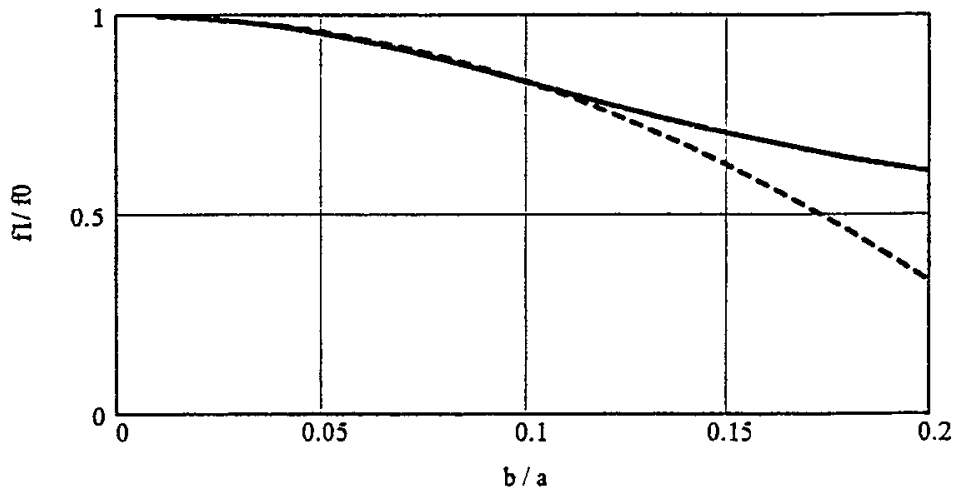

Exact

Approximate

(c) Epsr $=10$

Fig.2 


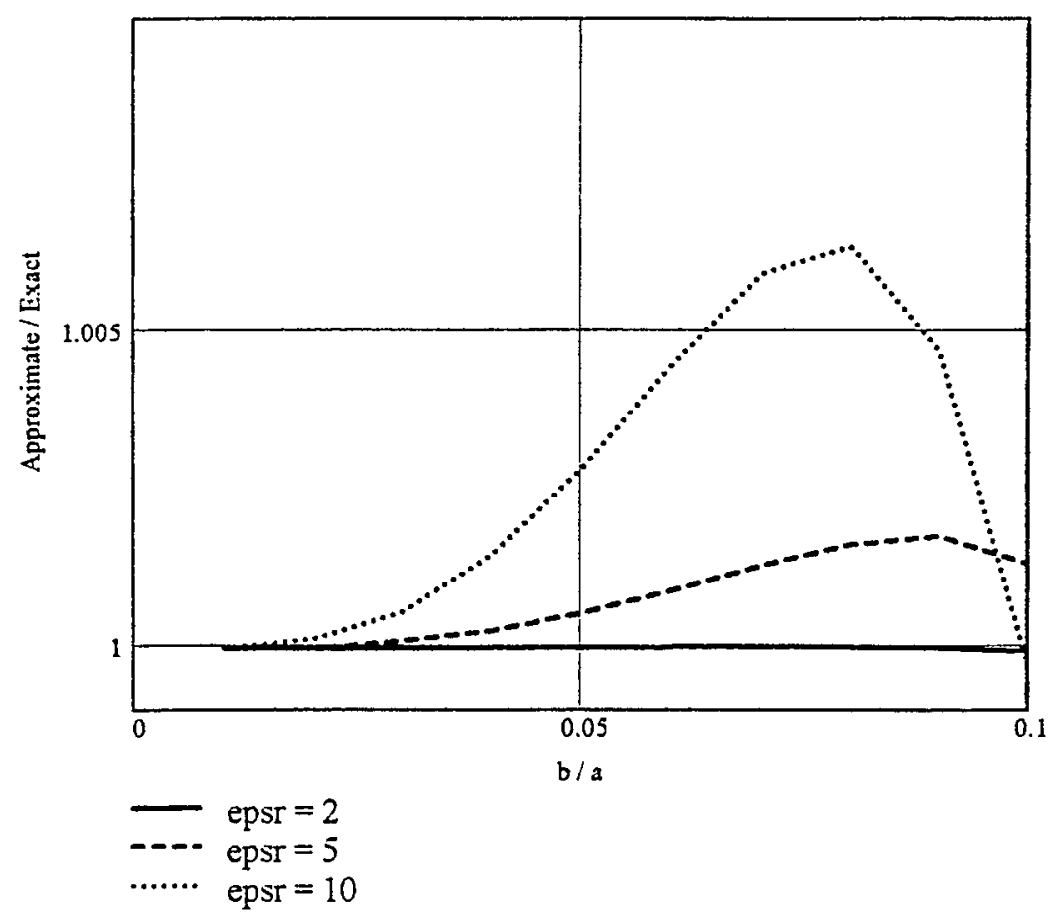

(a)

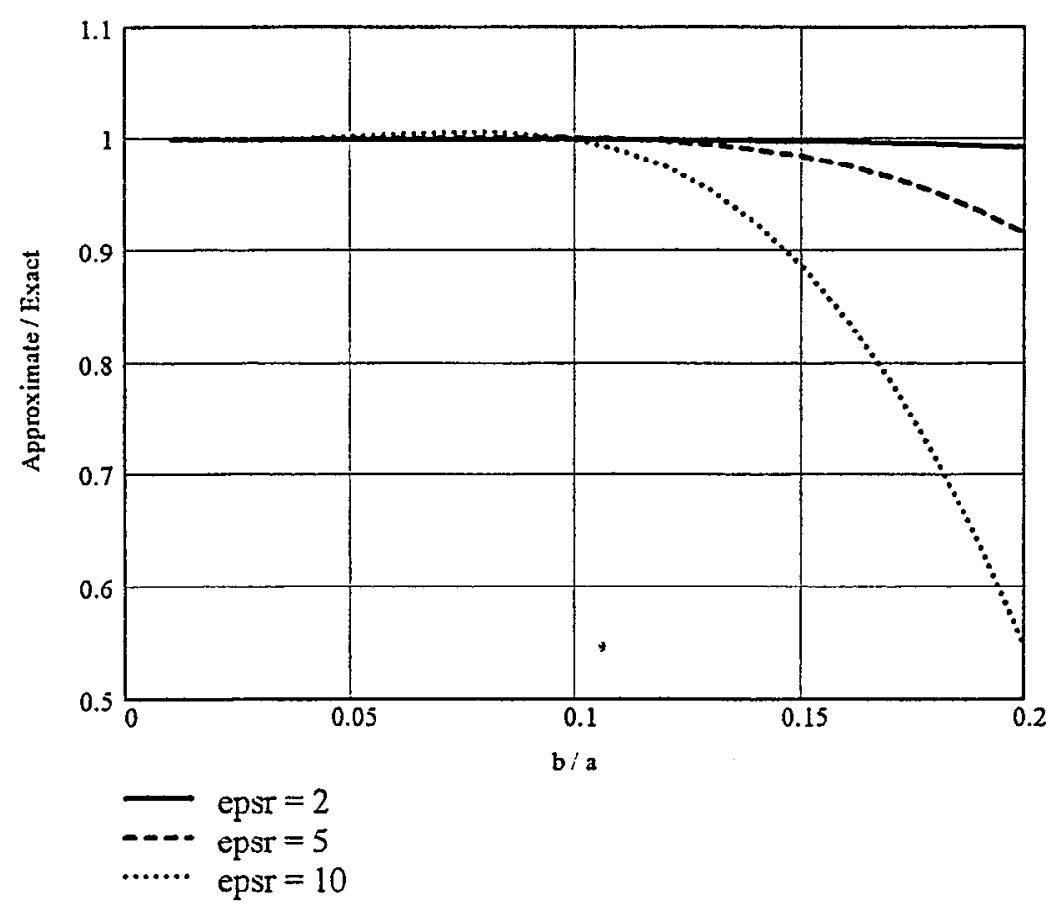

(b)

Fig.3 


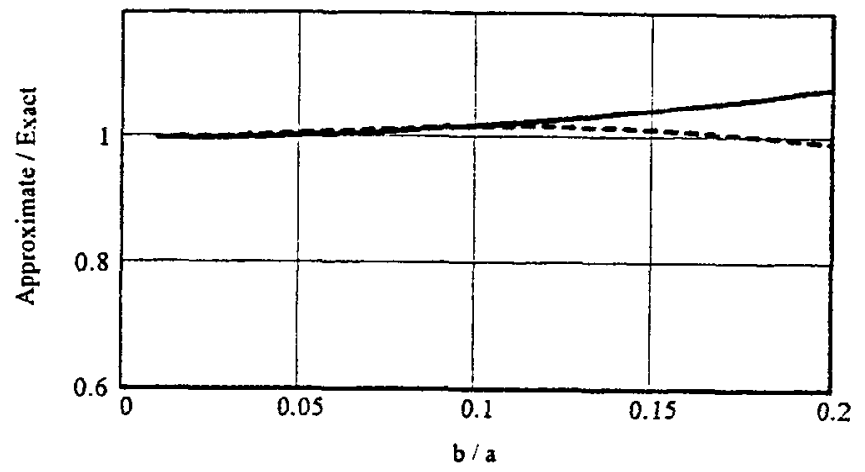

- Numerator

-... Denominator

(a) $\mathrm{Epst}=2$

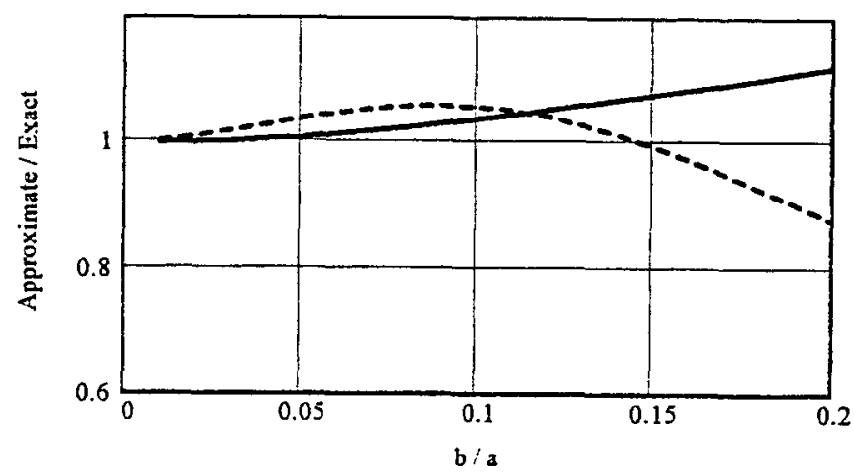

Numerator

-..- Denominator

(b) $E p s r=5$

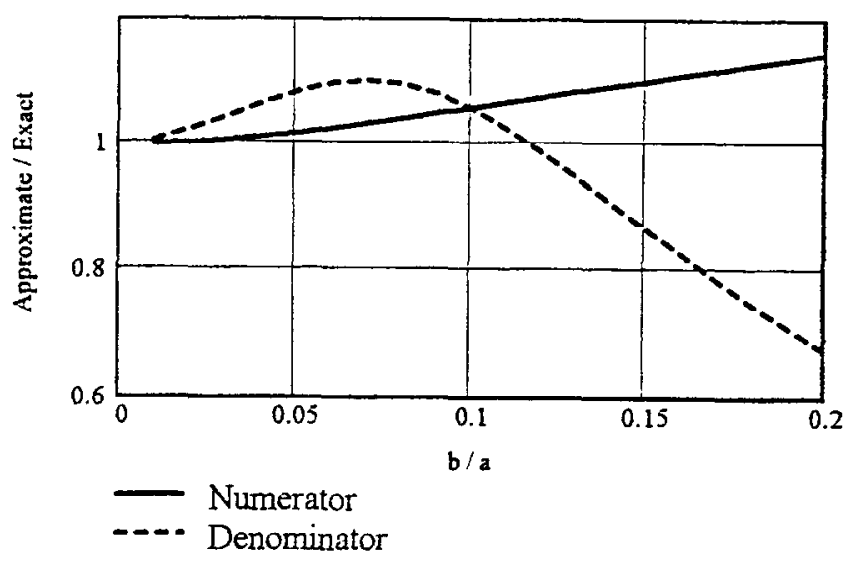

(c) $\mathrm{Epsr}=10$

Fig.4 


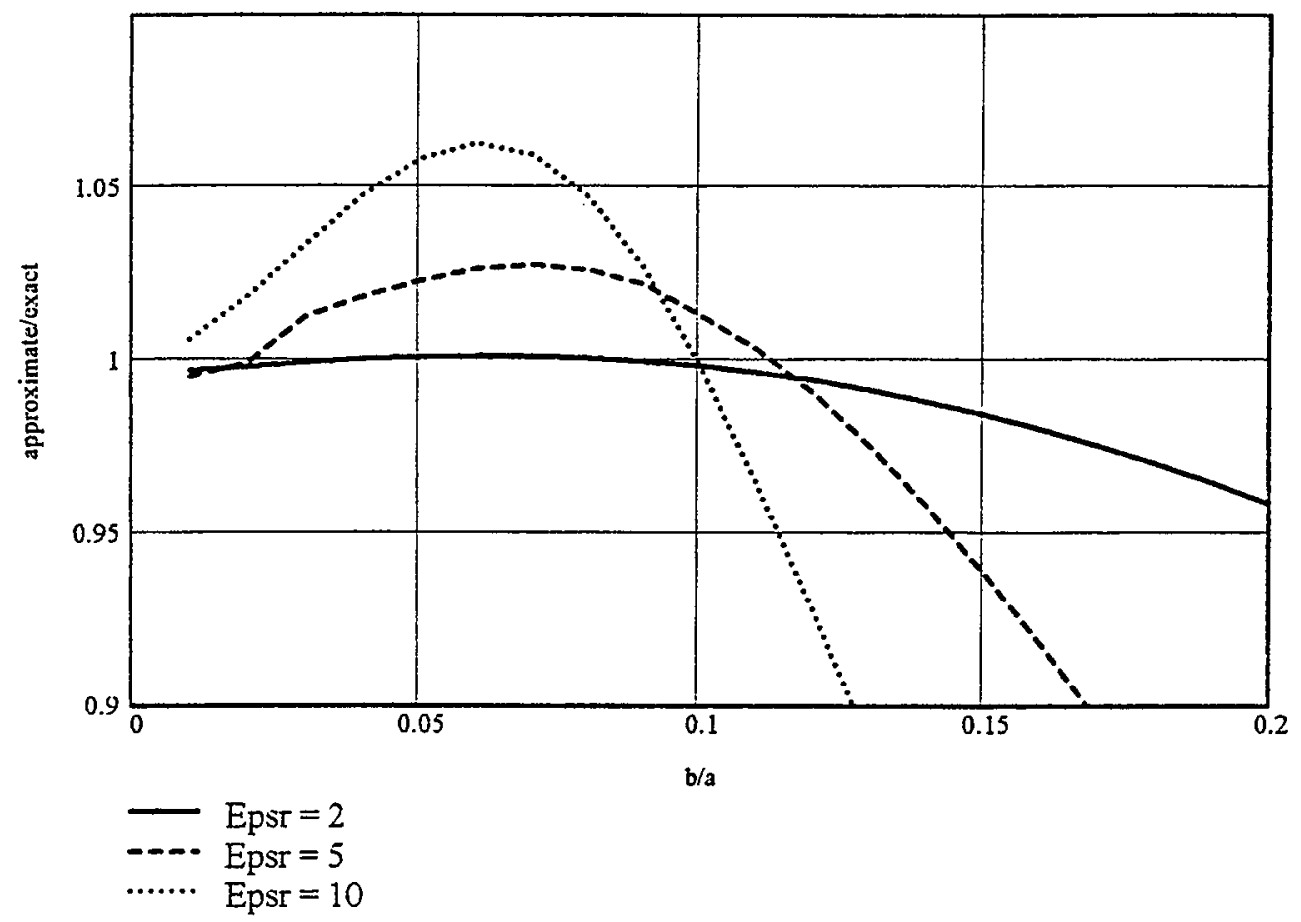

Fig.5 


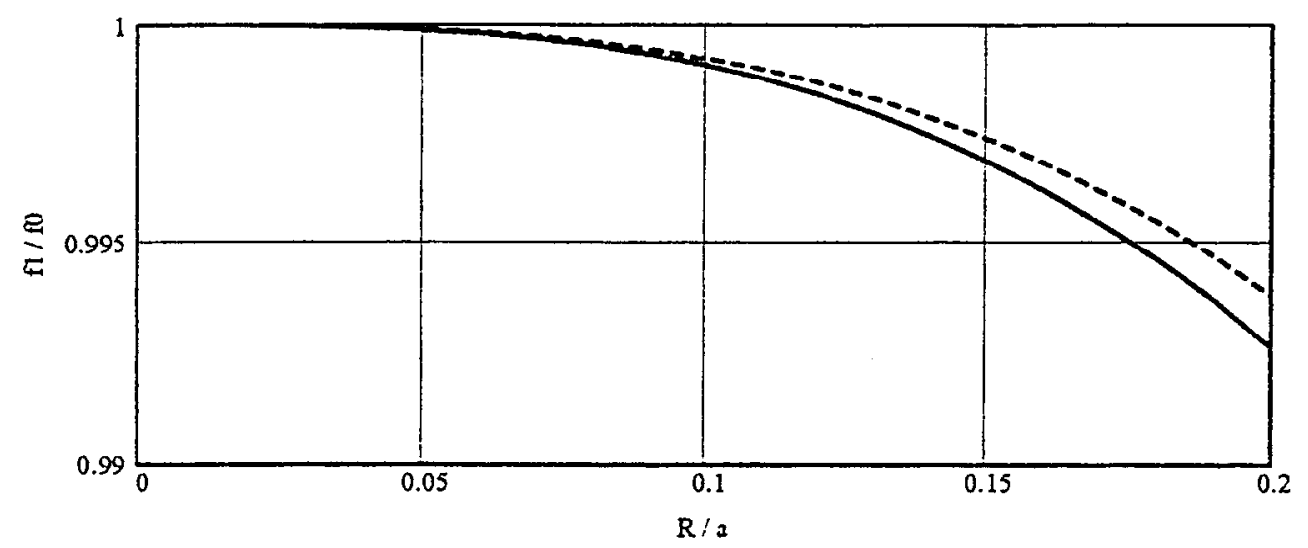

(a) $\varepsilon_{r}=2$

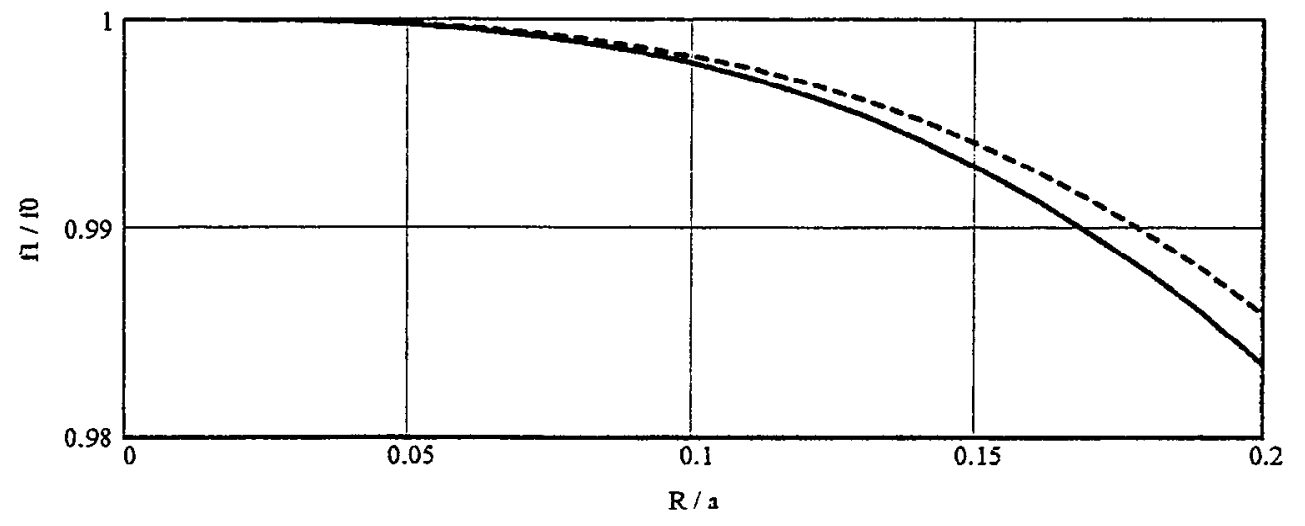

(b) $\varepsilon_{r}=5$

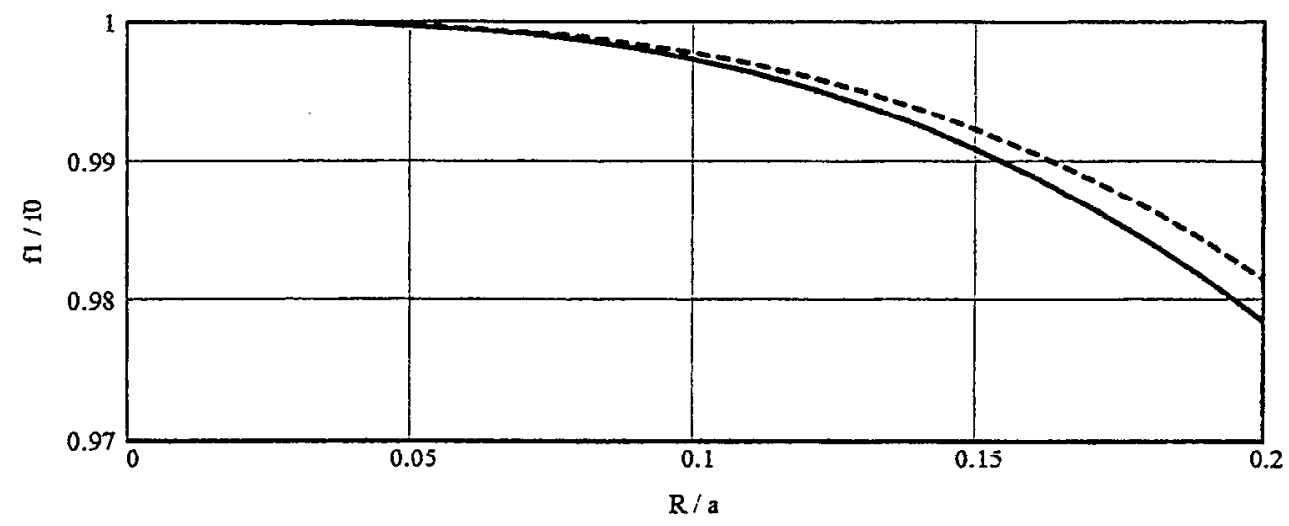

-.. Exact

(c) $\varepsilon_{r}=10$

Fig.6 


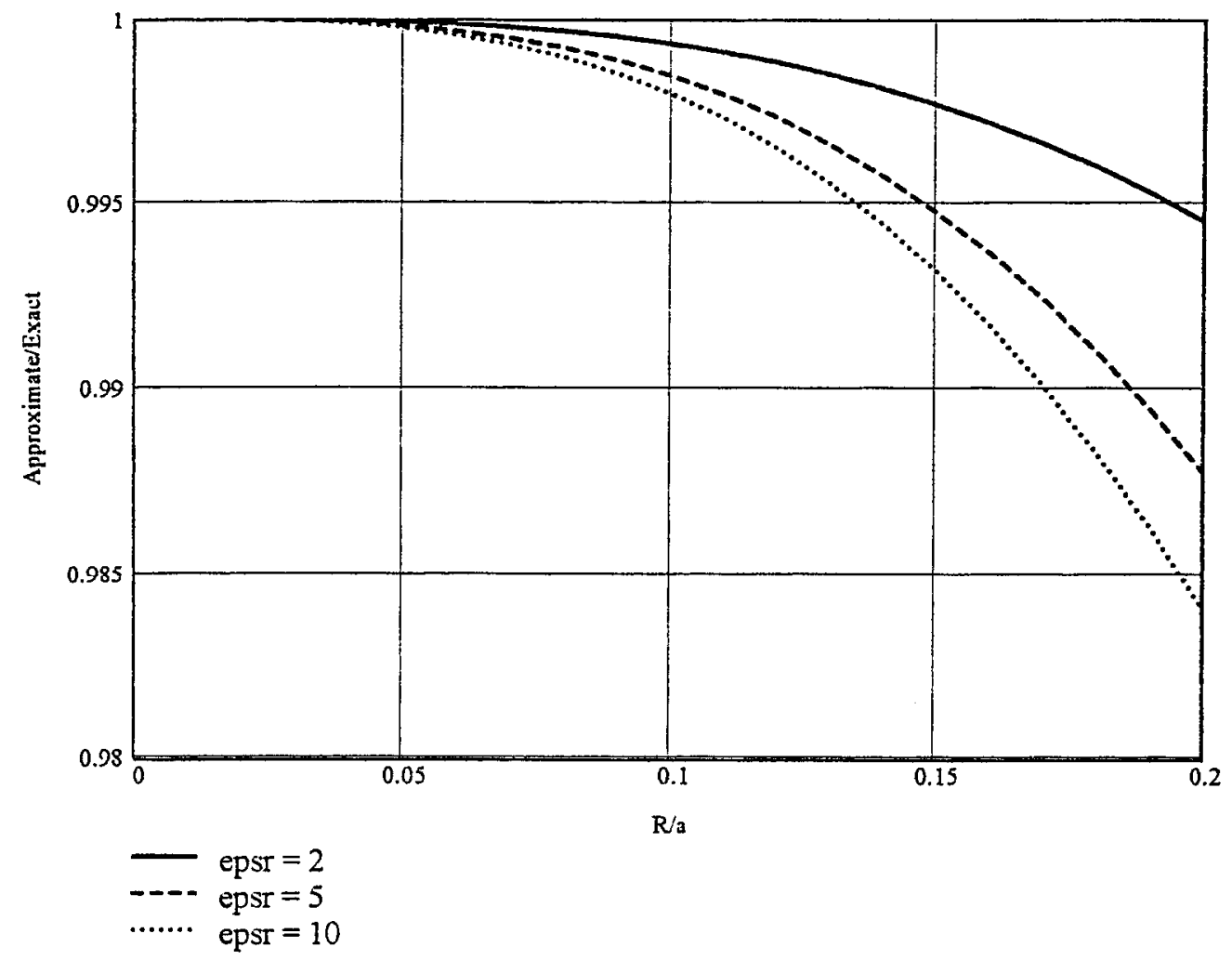

Fig.7 